\title{
The Effect of Parathyroid Gland Autotransplantation on Hypoparathyroidism After Thyroid Surgery for Papillary Thyroid Carcinoma
}

\author{
Bin Wang ${ }^{1, *}$ \\ Chun-Rong Zhu ${ }^{2, *}$ \\ Xin-Min Yao' \\ Jian $\mathrm{Wu} \mathbb{D}^{\prime}$
}

'Department of Thyroid and Breast Surgery, Chengdu Third People's Hospital, Chengdu, Sichuan Province, People's Republic of China; ${ }^{2}$ Department of Chemistry, School of Basic Medical Science, North Sichuan Medical College, Nanchong, Sichuan Province, People's Republic of China

*These authors contributed equally to this work
Correspondence: Jian Wu

Department of Thyroid and Breast Surgery, Chengdu Third People's Hospital,

No. 82, Qinglong Street, Chengdu, 61003I, Sichuan Province, People's

Republic of China

$\mathrm{Tel} / \mathrm{Fax}+862867566092$

Email wo_doctor@I63.com
Purpose: There are some controversies over the relationship between parathyroid gland autotransplantation and permanent hypoparathyroidism. This study aimed to explore the relationship between parathyroid gland autotransplantation and postoperative hypoparathyroidism.

Patients and Methods: We performed a retrospective analysis of patients who underwent initial thyroid surgery for papillary thyroid carcinoma from January 2014 to December 2018. Patients were divided into 4 groups according to the number of autotransplanted parathyroid glands (group 1: 0 autotransplanted parathyroid gland, group 2: 1 autotransplanted parathyroid gland, group 3: 2 autotransplanted parathyroid glands, group 4: 3 autotransplanted parathyroid glands). Clinical data were analyzed among the four groups.

Results: The more parathyroid glands were transplanted, the higher the incidence of immediate hypoparathyroidism was (group 1: $32.9 \%$, group 2: $52.9 \%$, group 3: $65.8 \%$, group 4: $82.4 \%$; $\mathrm{P}_{\text {group } 1 \text { vs group } 2}<0.001, \mathrm{P}_{\text {group } 2 \text { vs group } 3}=0.012, \mathrm{P}_{\text {group } 3 \text { vs group } 4}=0.17$ ). Parathyroid gland autotransplantation did not affect the incidence of permanent hypoparathyroidism (group 1: 1.4\%, group 2: $1.3 \%$, group 3: $0.9 \%$, group 4: $0 \% ; \mathrm{P}_{\text {group } 1 \text { vs group } 2}>0.99, \mathrm{P}_{\text {group } 2 \text { vs group } 3}>0.99$, $\mathrm{P}_{\text {group } 3 \text { vs group } 4}>0.99$ ).

Conclusion: The number of autotransplanted parathyroid glands was positively associated with the incidence of postoperative immediate hypoparathyroidism. Parathyroid gland autotransplantation was not associated with permanent hypoparathyroidism.

Keywords: parathyroid gland, autotransplantation, hypoparathyroidism, thyroid surgery

\section{Introduction}

Surgery was widely accepted as the primary therapy method for thyroid carcinoma. ${ }^{1,2}$ Among the complications of thyroid surgery, postoperative hypoparathyroidism was the most common. ${ }^{3,4}$ The incidence of transient hypoparathyroidism ranged from $10.8 \%$ to $64.4 \%$, and the incidence of permanent hypoparathyroidism varied from 0 to $31.1 \%$ across different researches. ${ }^{5-8}$ Hypoparathyroidism was the consequence of the reduction of the functional parathyroid parenchyma for mechanical or thermal trauma, devascularization, and inadvertent resection. ${ }^{9-11}$ Transient hypoparathyroidism may prolong hospitalization and increase the overall costs, and permanent hypoparathyroidism will severely affect the quality of life. . $^{9,13}$

In order to protect the parathyroid function, meticulous capsule dissection was used to preserve parathyroid glands and their blood supply, and autotransplantation was performed to save the function of parathyroid glands that were not able to be 
preserved in site. ${ }^{14,15}$ In recent years, routine parathyroid gland autotransplantation (PGA) was suggested to prevent permanent hypoparathyroidism because of its' predictable outcome. ${ }^{16,17}$ However, some studies discovered that PGA increased the risk of transient hypoparathyroidism and had no effects on permanent hypoparathyroidism. ${ }^{18,19}$ While some researchers indicated the incidence of hypoparathyroidism was associated with the number of autotransplanted parathyroid glands (APGs). ${ }^{10,13,20}$ In consideration of these controversies, we conducted the study to explore the relationship between PGA and postoperative hypoparathyroidism, investigate the influence of the number of APG in hypoparathyroidism, and identify the risk factors for postoperative hypoparathyroidism.

\section{Patients and Methods}

We performed a retrospective analysis of patients who underwent thyroid surgery for papillary thyroid carcinoma at our institution from January 2014 to December 2018. Patients who met all the following criteria were included: 1) patients who underwent the initial thyroid surgery; 2) the minimal surgical extent consisted of total thyroidectomy (TT) and bilateral central lymph node dissection (BCND). The exclusion criteria included: 1) patients who had undergone neck surgery; 2) patients who had received neck radiation therapy; 3) patients who suffered from parathyroid diseases at the same time; 4) patients whose preoperative serum parathyroid hormone (PTH) and/or calcium was abnormal; 5) patients who received calcium supplementation for other causes; 6) patients with the incomplete medical record; 7) patients who lost to follow-up in 6 months. The study was approved by the Medical Ethics Committee of Chengdu Third People's Hospital. And informed consent about the application of clinical data to medical research was obtained from all the subjects.

The surgical procedures were the same as those described by $\mathrm{Su}$ et al. ${ }^{21}$ Two professional thyroid surgeons (Wu J and Yao X) performed all procedures. Every parathyroid gland and its' blood supply were attempted to identify and preserve during surgery. Excisional thyroid and central tissue were routinely examined for any potential inadvertently excisional parathyroid glands. When a parathyroid gland was non-viable or found in the intraoperative specimens, it was fragmented into 1-mm pieces and inserted into 3-4 muscular pockets in the contralateral sternocleidomastoid muscle after histological confirmation by intraoperative frozen biopsy. The inadvertently excisional parathyroid gland (IEPG) was defined as the presence of parathyroid tissue in the postoperative specimens according to the pathologist.

Standardized perioperative management was applied to all patients with thyroid carcinoma. Neck ultrasound, laryngoscopy, thyroid function, serum PTH and calcium were routine preoperative assessments. Neck computerized tomography was used when the tumor was clinical suspicion for the advanced stage. Serum PTH and calcium were measured at 1 day, 1 month, 3 months, 6 months postoperatively. Intravenous calcium supplementation (calcium gluconate $10 \%, 20 \mathrm{~mL}$ ) was routinely administered at the end of surgery and 1 day postoperatively. After that, oral calcium was administered according to the postoperative serum PTH and symptoms. In these patients with mild hypoparathyroidism (PTH: $1.0 \sim 1.6 \mathrm{pmol} / \mathrm{l}$ ), oral calcium supplementation of $1200 \mathrm{mg} /$ day was prescribed. As for patients with medium hypoparathyroidism (PTH: $0.5 \sim 1.0$ $\mathrm{pmol} / \mathrm{l}$ ), the calcium of $1200 \mathrm{mg} /$ day and calcitriol of 1.0 ug/day were taken. And calcium of $1800 \mathrm{~g} /$ day and calcitriol $1.5 \mathrm{ug} /$ day were administered to the patients with severe hypoparathyroidism $(\mathrm{PTH}<0.5 \mathrm{pmol} / \mathrm{l})$. If patients suffered from mild symptoms, such as numbness of fingers, an upgraded level of strategy of calcium supplementation based on the level of serum PTH would be taken. While, intravenous calcium supplement was administered to the patients with severe symptoms, for example, spasms. Patients were discharged when their serum calcium was normal (2.1-2.7 $\mathrm{mmol} / \mathrm{l})$.

Hypoparathyroidism was defined as a serum PTH level of less than $1.6 \mathrm{pmol} / \mathrm{l}$ (normal range: 1.6-6.9 pmol/L) or presence of symptoms requiring calcium supplementation regardless of the PTH values. Hypocalcemia was defined as the less normal level in serum calcium (normal range: 2.1-2.7 mmol/1). Postoperative immediate hypoparathyroidism was defined as hypoparathyroidism at 1 day postoperatively. Protracted hypoparathyroidism was defined when hypoparathyroidism occurred at 1 month postoperatively. It was regarded as permanent hypoparathyroidism when hypoparathyroidism lasted for more than 6 months.

The collected data included the following information: the demographic characteristics, preoperative assessment, details of surgical extent, number of the APG and IEPG, pathologic report, the incidence of postoperative hypoparathyroidism, preoperative and postoperative serum PTH and calcium, and tumor characteristics. And according to the number of APG, patients were divided into 4 groups: group 1 (0 APG), group 2 (1 APG), group 3 (2 APGs), 
group 4 (3 APGs). The number of the parathyroid gland in site (ISPG) resulted from the formula: 4 minus the number of APG minus the number of IEPG.

The statistical analyses were conducted with SPSS version 23.0 software (SPSS Inc, Chicago, IL). Continuous data were expressed as mean \pm standard deviation (SD). Student's $t$-test or Mann-Whitney test was used for continuous variables, and Pearson's Chi-square test or Fisher's exact test was used for categorical variables. When the variables were potentially statistically significant in univariate analysis $(\mathrm{p}<0.1)$, they were analyzed by multivariable statistical analysis with binary logistic regression analysis to identify the risk factors for postoperative immediate and permanent hypoparathyroidism. Statistical significance was set at $\mathrm{P}<0.05$.

\section{Results}

A total of 658 patients were included in the retrospective cohort study. Among them, 216, 314, 111, and 17 patients underwent autotransplantation of $0,1,2$, and 3 parathyroid glands, respectively. There were no significant differences in the demographic characteristics (age, gender, body mass index), comorbidities (hypertension, diabetes, hyperthyroidism, hypothyroidism, and nodular goiter), preoperative serum PTH and calcium, tumor characteristics, and postoperative other complications (transient hoarseness, wound infection, postoperative bleeding, and chylous fistula) between group 1 and group 2, between group 2 and group 3, between group 3 and group 4 (Table 1). The gross extrathyroidal extension of the tumor, use of carbon nanoparticles, and more lymph nodes $(>10)$ were dissected in the central zone were associated with PGA $(\mathrm{P}=0.037, \mathrm{P}<$ $0.001, \mathrm{P}<0.001$, respectively; Table 1).

As shown in Table 2, the more parathyroid glands were transplanted, the lower level the serum PTH at 1 day postoperatively was (group 1: $2.36 \pm 1.38$, group 2: $1.79 \pm$ 1.18, group 3: $1.44 \pm 0.95$, group 4: $1.1 \pm 0.81$; $\mathrm{P}_{\text {group } 1 \text { vs group } 2<0.001,} \mathrm{P}_{\text {group } 2}$ vs group $3=0.002$, $\mathrm{P}_{\text {group } 3 \text { vs group } 4}=0.16$ ), the higher the incidence of immediate hypoparathyroidism become (group 1: 32.9\%, group 2: $52.9 \%$, group 3: $65.8 \%$, group 4: $82.4 \%$; $\mathrm{P}_{\text {group } 1 \text { vs group } 2}<0.001, \mathrm{P}_{\text {group } 2 \text { vs group } 3}=0.012$, $\mathrm{P}_{\text {group } 3}$ vs group $4=0.17$ ), and the longer the stay in hospital got (group 1: $4.2 \pm 2.2$, group 2: $4.9 \pm 2.9$, group 3: $5.8 \pm$

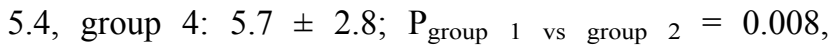
$\mathrm{P}_{\text {group } 2 \text { vs group } 3}=0.039, \mathrm{P}_{\text {group } 3}$ vs group $4=0.91$ ). However, PGA did not affect the incidence of protracted hypoparathyroidism (group 1: $5.1 \%$, group 2: $6.4 \%$, group

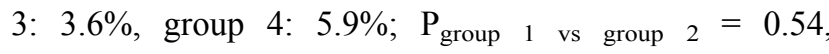
$\mathrm{P}_{\text {group } 2 \text { vs group } 3}=0.28, \mathrm{P}_{\text {group } 3}$ vs group $4=0.52$; Table 2) and permanent hypoparathyroidism (group 1: $1.4 \%$, group $2: 1.3 \%$, group $3: 0.9 \%$, group $4: 0 \%$;

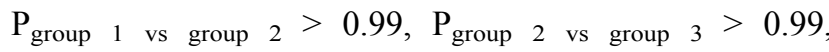
$\mathrm{P}_{\text {group } 3 \text { vs group } 4}>0.99$; Table 2 ).

Table 3 showed the details of the different management of parathyroid glands. A total of 587 parathyroid glands were autotransplanted among 442 patients, and 83 parathyroid glands were inadvertently resected among 80 patients. There were significant differences in the incidences of postoperative immediate hypoparathyroidism among patients with different numbers of APG when they did not undergo inadvertent parathyroid gland resection (0 APG: 28.8\%, 1 APG 51.1\%, 2 APG 63.3\%, 3 APG $82.4 \%, \mathrm{P}<0.001$; Table 3$)$. When one parathyroid gland was inadvertently resected, no significant differences in the incidence of postoperative immediate, protracted and permanent hypoparathyroidism were found among patients with different numbers of APG. When patients did not undergo PGA, the incidences of postoperative immediate hypoparathyroidism were significantly different among patients with different numbers of IEPG (0 IEPG:28.8\%, 1 IEPG: $59.1 \%$, 2 IEPGs: $100 \%, P=0.001$; Table 3), while differences were not found in the incidence of protracted and permanent hypoparathyroidism. There also were no significant differences in incidence of postoperative immediate, protracted and permanent hypoparathyroidism between patients with and without one IEPG after undergoing PGA.

The analyses of potential risk factors for postoperative immediate and permanent hypoparathyroidism were showed in Table 4. Gender, diabetes, hyperthyroidism, preoperative lymphadenectasis in the central zone, surgical extent, PGA, and inadvertently resected parathyroid gland might be associated with postoperative immediate hypoparathyroidism. And use of carbon nanoparticles and inadvertently resected parathyroid glands might affect permanent hypoparathyroidism. Multivariate analysis demonstrated that female gender $(\mathrm{OR}=1.45 ; 95 \%$ CI $1.02-2.06 ; \mathrm{p}=0.038)$, PGA (OR $=2.74 ; 95 \%$ CI $1.94-3.87 ; \mathrm{p}<0.001)$ and inadvertently resected parathyroid gland $(\mathrm{OR}=2.02 ; 95 \% \mathrm{CI} 1.23-3.32 ; \mathrm{p}=0.006)$ were independent risk factors for postoperative immediate hypoparathyroidism, and nonuse of carbon nanoparticles $(\mathrm{OR}=0.23 ; 95 \%$ CI $0.05-0.99 ; \mathrm{p}=0.049)$ and inadvertently removed parathyroid gland $(\mathrm{OR}=4.36$; 
Table I The Characteristics Among 4 Groups with Different Number of Autotransplanted Parathyroid Gland

\begin{tabular}{|c|c|c|c|c|c|c|c|}
\hline Variables & $\begin{array}{l}\text { Group I } \\
(2 \mid 6)\end{array}$ & $\begin{array}{l}\text { Group } 2 \\
(3 \mid 4)\end{array}$ & $\begin{array}{l}\text { Group } 3 \\
(I I I)\end{array}$ & $\begin{array}{l}\text { Group } 4 \\
\text { (I7) }\end{array}$ & $\mathbf{P}_{\text {Ivs2 }}$ & $\mathbf{P}_{2 v s 3}$ & $\mathbf{P}_{3 \mathrm{vs} 4}$ \\
\hline Gender (F/M) & $160 / 56$ & $219 / 95$ & $77 / 34$ & $12 / 5$ & 0.28 & 0.94 & 0.92 \\
\hline Age (years) & $41.8 \pm 13.1$ & $41.3 \pm 12.5$ & $42.1 \pm 12.2$ & $38.8 \pm 11.3$ & 0.64 & 0.54 & 0.3 \\
\hline BMI $\left(\mathrm{kg} / \mathrm{m}^{2}\right)$ & $22.9 \pm 3.4$ & $23.4 \pm 3.5$ & $23.1 \pm 3.4$ & $23.2 \pm 4.3$ & 0.09 & 0.42 & 0.89 \\
\hline Hypertension & 21 & 39 & 12 & 1 & 0.34 & 0.65 & 0.85 \\
\hline Diabetes & 5 & 11 & 2 & 2 & 0.43 & 0.57 & 0.09 \\
\hline Hyperthyroidism & 10 & 12 & 2 & 0 & 0.65 & 0.47 & $>0.99$ \\
\hline Hypothyroidism & 7 & 4 & 1 & 0 & 0.21 & $>0.99$ & $>0.99$ \\
\hline Hashimoto's Thyroiditis & 65 & 96 & 36 & 7 & 0.91 & 0.72 & 0.48 \\
\hline Nodular goiter & 117 & 176 & 63 & 12 & 0.67 & 0.9 & 0.28 \\
\hline Preoperative serum PTH (pmol/l) & $5.34 \pm 1.94$ & $5.77 \pm 2.22$ & $5.72 \pm 2.13$ & $5.47 \pm 2.03$ & 0.15 & 0.83 & 0.66 \\
\hline Preoperative serum calcium $(\mathrm{mmol} / \mathrm{l})$ & $2.33 \pm 0.1$ & $2.34 \pm 0.36$ & $2.32 \pm 0.11$ & $2.31 \pm 0.13$ & 0.68 & 0.66 & 0.68 \\
\hline Carbon nanoparticles & 176 & 289 & 100 & 13 & $<0.001$ & 0.53 & 0.22 \\
\hline $\begin{array}{l}\text { Preoperative lymphadenectasis in the central } \\
\text { zone }\end{array}$ & 52 & 102 & 49 & 8 & 0.14 & 0.027 & 0.82 \\
\hline Largest size of lymph nodes $(\mathrm{mm})$ & $12.8 \pm 5.3$ & $12 \pm 4.6$ & $13.5 \pm 6.4$ & $|3.| \pm 6 . \mid$ & 0.38 & 0.14 & 0.87 \\
\hline Surgical extent & & & & & 0.12 & 0.18 & 0.68 \\
\hline $\mathrm{TT}+\mathrm{BCND}$ & 179 & 237 & 75 & 12 & & & \\
\hline TT+BCND+ULND & 32 & 65 & 28 & 5 & & & \\
\hline $\mathrm{TT}+\mathrm{BCND}+\mathrm{BLND}$ & 5 & 12 & 8 & 0 & & & \\
\hline Largest tumor size $(\mathrm{mm})$ & $16 \pm 13.2$ & $15.8 \pm 9.9$ & $16.8 \pm 10.8$ & $\mid 4.1 \pm 5.0$ & 0.88 & 0.38 & 0.1 \\
\hline Tumor location (upper/middle/lower/isthmus) & $58 / 84 / 62 / 12$ & $87 / \mid 24 / 82 / 21$ & $22 / 50 / 24 / 15$ & $6 / 5 / 3 / 3$ & 0.89 & 0.051 & 0.55 \\
\hline Bilateral lobe tumors & 50 & 87 & 31 & I & 0.24 & 0.96 & 0.1 \\
\hline Multifocal tumors & 55 & 100 & 34 & 4 & 0.11 & 0.81 & 0.55 \\
\hline Gross extrathyroidal extension & 26 & 59 & 30 & 2 & 0.037 & 0.07 & 0.29 \\
\hline Number of lymph nodes in the central zone & $12.8 \pm 6.3$ & $13.8 \pm 6.7$ & $14.6 \pm 7.0$ & $14.9 \pm 7.2$ & 0.09 & 0.28 & 0.86 \\
\hline$\leq 10 />10$ & $126 / 90$ & $88 / 226$ & $29 / 82$ & $2 / 15$ & $<0.001$ & 0.7 & 0.33 \\
\hline $\begin{array}{l}\text { Number of metastatic lymph nodes in central } \\
\text { zone }\end{array}$ & $3.5 \pm 3.9$ & $4.1 \pm 4.6$ & $4.6 \pm 4.7$ & $4.4 \pm 4.7$ & 0.13 & 0.27 & 0.86 \\
\hline$\leq 5 />5$ & $154 / 62$ & $204 / 110$ & $67 / 44$ & $13 / 4$ & 0.13 & 0.39 & 0.2 \\
\hline $\begin{array}{l}\text { Pathological T classification (TI/T2/T3a/T3b/ } \\
\text { T4) }\end{array}$ & $160 / 24 / 6 / 23 / 3$ & $\begin{array}{l}210 / 39 / 6 / 40 / \\
19\end{array}$ & $65 / 15 / 1 / 19 / 11$ & $13 / 2 / 0 / 2 / 0$ & 0.07 & 0.36 & 0.12 \\
\hline Pathological N classification (No/NI) & $58 / 158$ & $75 / 239$ & $22 / 89$ & $4 / 13$ & 0.44 & 0.38 & 0.98 \\
\hline AJCC stage $(\mathrm{I} \sim \mathrm{II} / \mathrm{III} \sim \mathrm{IV})$ & $215 / 1$ & $308 / 6$ & $10 / 1$ & $17 / 0$ & 0.25 & 0.78 & $>0.99$ \\
\hline Inadvertently resected PG & 25 & 42 & 13 & 0 & 0.54 & 0.65 & 0.29 \\
\hline Transient hoarseness & 5 & 9 & 6 & 0 & 0.7 & 0.24 & 0.65 \\
\hline Wound infection & 4 & 10 & 5 & 0 & 0.34 & 0.73 & $>0.99$ \\
\hline Postoperative bleeding & 2 & 4 & I & 0 & $>0.99$ & $>0.99$ & $>0.99$ \\
\hline Chylous fistula & 0 & 2 & 4 & 0 & 0.51 & 0.043 & $>0.99$ \\
\hline
\end{tabular}

Notes: $P_{\text {Ivs2 }}$, Group I vs Group 2; $P_{2 \text { vs } 3}$, Group 2 vs Group 3; $P_{3 v s 4}$, Group 3 vs Group 4.

Abbreviations: F, female; $M$ male; BMI body mass index; PTH parathyroid hormone; TT total thyroidectomy; BCND bilateral central lymph node dissection; ULND unilateral neck lymph node dissection (compartments II-V); BLND bilateral neck lymph node dissection (compartments II-V); PG, parathyroid gland.

95\% CI 1.01-18.79; $\mathrm{p}=0.049)$ were independent risk factors for permanent hypoparathyroidism (Table 5).

\section{Discussions}

PGA is the exclusive way to restore the function of the parathyroid gland that could not be preserved in site. However, some researchers found that the function of the
APG was not always able to recover to the preoperative level. ${ }^{22-24}$ Even so, some surgeons discovered PGA could reduce the incidence of permanent hypoparathyroidism and thus suggested routinely performed it. ${ }^{16,17}$ However, some other studies suggested that PGA did not affect, even increase the incidence of permanent hypoparathyroidism. ${ }^{8,25}$ 
Table 2 The Influence of Different Numbers of Autotransplanted Parathyroid Gland

\begin{tabular}{|c|c|c|c|c|c|c|c|}
\hline Variables & $\begin{array}{l}\text { Group I } \\
(2 \mid 6)\end{array}$ & $\begin{array}{l}\text { Group } 2 \\
(3 \mid 4)\end{array}$ & $\begin{array}{l}\text { Group } 3 \\
(\mathrm{III})\end{array}$ & $\begin{array}{l}\text { Group } 4 \\
\text { (I7) }\end{array}$ & $\mathbf{P}_{\text {Ivs2 }}$ & $\mathbf{P}_{2 \mathrm{vs3}}$ & $\mathbf{P}_{3 \mathrm{vs} 4}$ \\
\hline \multicolumn{8}{|l|}{ Postoperative PTH (pmol/l) } \\
\hline I day & $2.36 \pm 1.38$ & $1.79 \pm 1.18$ & $1.44 \pm 0.95$ & $1.1 \pm 0.8 \mathrm{I}$ & $<0.001$ & 0.002 & 0.16 \\
\hline I month & $3.79 \pm 1.75$ & $3.6 \pm 1.59$ & $3.73 \pm|.5|$ & $2.85 \pm 1.32$ & 0.24 & 0.51 & 0.049 \\
\hline 6 months & $3.99 \pm 1.73$ & $3.81 \pm 1.7$ & $3.8 \pm 1.23$ & $2.95 \pm 1.27$ & 0.54 & 0.95 & 0.2 \\
\hline $\begin{array}{l}\text { Postoperative immediate hypoparathyroidism } \\
(\%)\end{array}$ & 7I (32.9) & $163(51.9)$ & $73(65.8)$ & $14(82.4)$ & $<0.001$ & 0.012 & 0.17 \\
\hline Protracted hypoparathyroidism (\%) & II (5.I) & $20(6.4)$ & $4(3.6)$ & I (5.9) & 0.54 & 0.28 & 0.52 \\
\hline Permanent hypoparathyroidism (\%) & $3(1.4)$ & $4(1.3)$ & $\mathrm{I}(0.9)$ & $0(0)$ & $>0.99$ & $>0.99$ & $>0.99$ \\
\hline \multicolumn{8}{|l|}{ Postoperative serum calcium (mmol/l) } \\
\hline I day & $2.16 \pm 0.15$ & $2.11 \pm 0.15$ & $2.06 \pm 0.19$ & $2.08 \pm 0.1$ & $<0.001$ & 0.021 & 0.79 \\
\hline I month & $2.3 \pm 0.15$ & $2.29 \pm 0.19$ & $2.33 \pm 0.33$ & $2.24 \pm 0.1$ & 0.66 & 0.12 & 0.27 \\
\hline 6 months & $2.26 \pm 0.2$ & $2.23 \pm 0.18$ & $2.26 \pm 0.15$ & $2.26 \pm 0.13$ & 0.47 & 0.57 & 0.99 \\
\hline Postoperative immediate hypocalcemia (\%) & $69(31.9)$ & $146(46.5)$ & $56(50.5)$ & $8(47.1)$ & 0.001 & 0.47 & 0.8 \\
\hline Protracted hypocalcemia (\%) & $18(8.3)$ & $30(9.6)$ & $4(3.6)$ & I (5.9) & 0.63 & 0.047 & 0.52 \\
\hline Permanent hypocalcemia (\%) & $2(0.9)$ & $7(2.2)$ & I (0.9) & $0(0)$ & 0.41 & 0.62 & $>0.99$ \\
\hline Postoperative length of stay (days) & $4.2 \pm 2.2$ & $4.9 \pm 2.9$ & $5.8 \pm 5.4$ & $5.7 \pm 2.8$ & 0.008 & 0.039 & 0.91 \\
\hline
\end{tabular}

Abbreviation: PTH, parathyroid hormone.

Table 3 Comparison Among Groups with Different Management of Parathyroid Gland

\begin{tabular}{|c|c|c|c|c|}
\hline $\begin{array}{l}\text { The Number of } \\
\text { Parathyroid Glands }\end{array}$ & $\mathbf{N}$ & $\begin{array}{l}\text { Postoperative Immediate } \\
\text { Hypoparathyroidism (\%) }\end{array}$ & $\begin{array}{l}\text { Protracted } \\
\text { Hypoparathyroidism (\%) }\end{array}$ & $\begin{array}{l}\text { Permanent } \\
\text { Hypoparathyroidism (\%) }\end{array}$ \\
\hline 0 APG & 216 & 7I(32.9) & II (5.I) & $3(1.4)$ \\
\hline 4 ISPG & 191 & $55(28.8)$ & $9(4.7)$ & $2(1.0)$ \\
\hline 3 ISPG+I IEPG & 22 & $13(59.1)$ & $2(9.1)$ & $\mathrm{I}(4.5)$ \\
\hline 2 ISPG +2 IEPG & 3 & $3(100)$ & $0(0)$ & $0(0)$ \\
\hline$P_{\text {OAPG }}$ & & 0.001 & 0.63 & 0.41 \\
\hline I APG & 314 & $163(51.9)$ & $20(6.4)$ & $4(1.3)$ \\
\hline 3 ISPG & 272 & $139(51.1)$ & $18(6.6)$ & $3(1.1)$ \\
\hline 2 ISPG+I IEPG & 42 & $24(57.1)$ & $2(4.8)$ & $\mathrm{I}(2.4)$ \\
\hline$P_{I A P G}$ & & 0.47 & 0.91 & 0.44 \\
\hline 2 APG & 111 & $73(65.8)$ & $4(3.6)$ & $\mathrm{I}(0.9)$ \\
\hline 2 ISPG & 98 & $62(63.3)$ & $3(3.1)$ & $0(0)$ \\
\hline I ISPG+I IEPG & 13 & II (84.6) & $\mathrm{I}(7.7)$ & $\mathrm{I}(7.7)$ \\
\hline$P_{2 A P G}$ & & 0.21 & 0.40 & 0.12 \\
\hline 3 APG+I ISPG & 17 & I4(82.4) & $I(5.9)$ & $0(0)$ \\
\hline $\mathrm{P}_{4 \mathrm{PGs}}$ & & $<0.001$ & 0.57 & 0.74 \\
\hline$P_{3 P G s}$ & & 0.19 & 0.79 & 0.68 \\
\hline
\end{tabular}

Notes: $\mathrm{P}_{\mathrm{OAPG}}, \mathrm{P}$ value of comparison among rates of groups without autotransplanted parathyroid gland; $\mathrm{P}_{\mathrm{IAPG}}, \mathrm{P}$ value of comparison among rates of groups with $\mathrm{I}$ autotransplanted parathyroid gland; $P_{2 A P G}, P$ value of comparison among rates of groups with 2 autotransplanted parathyroid glands; $P_{4 P G s}, P$ value of comparison among rates of groups without inadvertently resected parathyroid gland; $\mathrm{P}_{3 \mathrm{PG}}, \mathrm{P}$ value of comparison among rates of groups with one inadvertently resected parathyroid gland. Abbreviation: APG autotransplanted parathyroid gland; ISPG parathyroid gland in site; IEPG inadvertently excisional parathyroid gland.

In the present study, use of carbon nanoparticles, gross extrathyroidal extension of the tumor, and more excisional lymph nodes $(>10)$ in the central zone increased the incidence of PGA. In recent decades, carbon nanoparticles has been used as a tracer to stain the thyroid and lymph system during thyroid surgery. ${ }^{26,27}$ Because it did not stain the parathyroid glands, carbon nanoparticles could help to identify them, which might assist to discover the parathyroid gland in the specimens during surgery and thus increase the number of $\mathrm{APG}^{26,27}$ Gross extrathyroidal 
Table 4 Univariate Analysis of Risk Factors for the Postoperative Immediate and Permanent Hypoparathyroidism

\begin{tabular}{|c|c|c|c|c|c|c|}
\hline \multirow[t]{2}{*}{ Variables } & \multicolumn{3}{|c|}{$\begin{array}{l}\text { Postoperative Immediate } \\
\text { Hypoparathyroidism }\end{array}$} & \multicolumn{3}{|c|}{ Permanent Hypoparathyroidism } \\
\hline & No (337) & Yes (32I) & $\mathbf{P}$ & No (650) & Yes (8) & $\mathbf{P}$ \\
\hline Gender (F/M) & $229 / 108$ & $239 / 82$ & 0.066 & $461 / 189$ & $7 / 1$ & 0.53 \\
\hline Age (years) & $41.8 \pm 12.5$ & $41.2 \pm 12.7$ & 0.50 & $41.5 \pm 12.5$ & $42.8 \pm 17.3$ & 0.78 \\
\hline BMI $\left(\mathrm{kg} / \mathrm{m}^{2}\right)$ & $23.2 \pm 3.5$ & $23.2 \pm 3.4$ & 0.78 & $23.2 \pm 3.4$ & $23.2 \pm 4.2$ & $>0.99$ \\
\hline Hypertension & 42 & 31 & 0.25 & 72 & 1 & $>0.99$ \\
\hline Diabetes & 6 & 14 & 0.054 & 20 & 0 & $>0.99$ \\
\hline Hyperthyroidism & 17 & 7 & 0.050 & 24 & 0 & $>0.99$ \\
\hline Hypothyroidism & 6 & 6 & 0.93 & 12 & 0 & $>0.99$ \\
\hline Hashimoto's Thyroiditis & 104 & 100 & 0.94 & 201 & 3 & 0.99 \\
\hline Nodular goiter & 183 & 185 & 0.39 & 364 & 4 & 0.74 \\
\hline Preoperative serum PTH (pmol/l) & $5.68 \pm 2.06$ & $5.48 \pm 2.19$ & 0.24 & $5.59 \pm 2.13$ & $5.17 \pm 1.93$ & 0.58 \\
\hline Preoperative serum calcium (mmol/l) & $2.32 \pm 0.12$ & $2.34 \pm 0.36$ & 0.18 & $2.33 \pm 0.27$ & $2.3 \pm 0.16$ & 0.78 \\
\hline Carbon nanoparticles & 302 & 276 & 0.15 & 573 & 5 & 0.062 \\
\hline Preoperative lymphadenectasis in the central zone & 96 & 115 & 0.044 & 207 & 4 & 0.48 \\
\hline Largest size of lymph nodes $(\mathrm{mm})$ & $12.6 \pm 5.5$ & $12.6 \pm 5.2$ & 0.90 & $12.5 \pm 5.3$ & $16 \pm 6.2$ & 0.20 \\
\hline Surgical extent & & & 0.055 & & & 0.29 \\
\hline $\mathrm{TT}+\mathrm{BCND}$ & 271 & 234 & & 500 & 5 & \\
\hline$T T+B C N D+U L N D$ & 57 & 71 & & 126 & 2 & \\
\hline TT+BCND+BLND & 9 & 16 & & 24 & 1 & \\
\hline Largest tumor size (mm) & $15.6 \pm 10.2$ & $16.4 \pm 12.1$ & 0.34 & $15.9 \pm 10.8$ & $26.1 \pm 27.4$ & 0.33 \\
\hline Tumor location (upper/middle/lower/isthmus) & $93 / 109 / 115 / 20$ & $81 / 119 / 91 / 30$ & 0.13 & $170 / 227 / 204 / 49$ & $4 / 1 / 2 / 1$ & 0.49 \\
\hline Bilateral lobe tumors & 80 & 89 & 0.24 & 168 & 1 & 0.65 \\
\hline Multifocal tumors & 94 & 99 & 0.41 & 192 & 1 & 0.51 \\
\hline Gross extrathyroidal extension & 56 & 61 & 0.42 & 115 & 2 & 0.94 \\
\hline Number of lymph nodes in the central zone & $13.6 \pm 6.7$ & $13.5 \pm 6.6$ & 0.86 & $13.6 \pm 6.6$ & $14.4 \pm 10.1$ & 0.74 \\
\hline$\leq 10 />10$ & $103 / 234$ & $95 / 226$ & 0.78 & $194 / 456$ & $4 / 4$ & 0.4 \\
\hline Number of metastatic lymph nodes in central zone & $3.8 \pm 4.4$ & $4.2 \pm 4.5$ & 0.27 & $4 \pm 4.4$ & $3.8 \pm 3.4$ & 0.89 \\
\hline$\leq 5 />5$ & $225 / 112$ & $213 / 108$ & 0.91 & $433 / 217$ & $5 / 3$ & $>0.99$ \\
\hline Pathological T classification (TI T2/T3 T4) & $277 / 60$ & $251 / 70$ & 0.2 & $523 / 127$ & $5 / 3$ & 0.41 \\
\hline Pathological N classification (pN0/pNI) & $89 / 248$ & $70 / 251$ & 0.17 & $158 / 492$ & $\mathrm{I} / 7$ & 0.72 \\
\hline AJCC stage $(|\sim| I / I I|\sim| V)$ & $331 / 6$ & $319 / 2$ & 0.29 & $642 / 8$ & $8 / 0$ & $>0.99$ \\
\hline PGA & 192 & 250 & $<0.001$ & 437 & 5 & 0.72 \\
\hline Inadvertently resected PG & 29 & 51 & 0.004 & 77 & 3 & 0.062 \\
\hline Transient hoarseness & 11 & 9 & 0.73 & 20 & 0 & $>0.99$ \\
\hline Wound infection & 10 & 9 & 0.9 & 19 & 0 & $>0.99$ \\
\hline Postoperative bleeding & 4 & 3 & $>0.99$ & 7 & 0 & $>0.99$ \\
\hline Chylous fistula & 3 & 3 & $>0.99$ & 6 & 0 & $>0.99$ \\
\hline
\end{tabular}

Abbreviations: F, female; M male; BMI body mass index; PTH parathyroid hormone; TT total thyroidectomy; BCND bilateral central lymph node dissection; ULND unilateral neck lymph node dissection (compartments II-V); BLND bilateral neck lymph node dissection (compartments II-V); PG, parathyroid gland.

extension of the tumor might lead to increased difficulty in preserving parathyroid glands in their site and protecting their blood supply. More excisional lymph nodes meant a larger surgery extent, which would be an adverse factor in protecting the parathyroid gland.

Several studies demonstrated that the value of serum PTH at 1 day postoperatively decreased and the incidence of postoperative transient hypoparathyroidism increased as the parathyroid gland was transplanted..$^{10,13,28}$ The current research was consistent with previous researches. It took at least 4 weeks for the APG to recover full function. ${ }^{29,30}$ So, the functional parathyroid gland tissue would reduce during this period when the number of APG increased. The number of APG did not significantly affect the incidence of postoperative protracted and permanent hypoparathyroidism. It might be the reason that almost all the patients had three or more functional parathyroid glands regardless of whether they were preserved in site or autotransplanted. 
Table 5 Multivariate Analysis of Risk Factors for Postoperative Immediate and Permanent Hypoparathyroidism

\begin{tabular}{|c|c|c|c|c|c|c|}
\hline \multirow[t]{2}{*}{ Variables } & \multicolumn{3}{|c|}{$\begin{array}{l}\text { Postoperative Immediate Hypoparathyroidism } \\
(G=866.1 \text { I 2,P<0.00I) }\end{array}$} & \multicolumn{3}{|c|}{$\begin{array}{l}\text { Permanent Hypoparathyroidism (G=79.766, } \\
\text { P<0.00I) }\end{array}$} \\
\hline & OR & $95 \% \mathrm{Cl}$ & $\mathbf{P}$ & OR & $95 \% \mathrm{Cl}$ & $\mathbf{P}$ \\
\hline Female & 1.45 & $1.02-2.06$ & 0.038 & & & \\
\hline Carbon nanoparticles & & & & 0.23 & $0.05-0.99$ & 0.049 \\
\hline PGA & 2.74 & $1.94-3.87$ & $<0.001$ & & & \\
\hline Inadvertently resected PG & 2.02 & $1.23-3.32$ & 0.006 & 4.36 & $1.01-18.79$ & 0.049 \\
\hline
\end{tabular}

Abbreviations: PGA, parathyroid gland autotransplantation; PG, parathyroid gland.

Patients with three or more ISPGs scarcely underwent permanent hypoparathyroidism, nor did patients with 3 or more APGs. 3,31

It was also controversial whether the parathyroid gland with potential injury of blood supply (parathyroid gland with some degree of discolored) should be preserved in site or autotransplanted. Su et al and Lorente-Poch et al indicated that when three parathyroid glands were preserved in site, the fourth parathyroid gland did not significantly affect the incidence of permanent hypoparathyroidism regardless of whether it was preserved in site or autotransplanted. ${ }^{11,15}$ This study suggested when the number of functional parathyroid glands was equal, no matter what method was used to protect their function (preserved in site or autotransplanted), the incidences of protracted and permanent hypoparathyroidism were similar. This phenomenon might attribute to the fact that at least one parathyroid gland was preserved in site and the number of functional parathyroid glands was more than three. Song and his colleagues demonstrated that one ISPG was sufficient to prevent permanent hypoparathyroidism. ${ }^{32}$

In the current study, the incidence of postoperative immediate hypoparathyroidism was $48.8 \%$, and the complication prolonged the postoperative length of stay $(5.5$ \pm 3.8 days vs $4.3 \pm 2.6$ days, $\mathrm{P}<0.001)$. Female gender was one of the risk factors for postoperative immediate hypoparathyroidism in the study, which had been reported in the previous study. ${ }^{33}$ Some studies suggested the proportions of parenchymal fat and stromal fat in parathyroid tissue were different between males and females. $^{34,35}$ And Dufour et al confirmed that the weight of the parathyroid gland in men was higher than that in women. ${ }^{35}$ Besides, PTH secretion was affected by sex steroids, which were different between males and females. $^{36,37}$ It was also speculated that the gender disparity might lead to the difference in regulators of monoclonal proliferation and mitosis of parathyroid tissue and the difference in the anatomy of the parathyroid gland. ${ }^{37,38}$ As for other risk factors for immediate hypoparathyroidism, PGA and inadvertently resected parathyroid gland both led to the reduction of postoperative realtime functional parathyroid parenchyma.

The study also suggested that the use of carbon nanoparticles was the protective factor for permanent hypoparathyroidism. Several studies indicated that carbon nanoparticles could assist to identify the parathyroid gland and decrease the incidence of the inadvertently resected parathyroid gland. ${ }^{26,27}$ Consistent with previous researches, inadvertently resected parathyroid gland increased the incidence of permanent hypoparathyroidism. ${ }^{39,40}$

There were several limitations in this study. First, the data were retrospectively analyzed, and some selective biases were inevitable. Second, the APG could not be evaluated objectively. Third, the sample size of the study, especially permanent hypoparathyroidism, was small. Research with larger sample size and objective evaluation indicators, such as parathyroid glands were transplanted in the forearm, was necessary to be conducted to verify the result in this study.

\section{Conclusions}

In conclusion, PGA increases the risk of postoperative immediate hypoparathyroidism, and the more parathyroid glands are transplanted, the more likely the immediate hypoparathyroidism occurs. Besides, female gender and inadvertently resected parathyroid gland may increase the risk of immediate hypoparathyroidism. PGA does not affect permanent hypoparathyroidism when three or more functional parathyroid glands are reserved regardless of whether they are 
preserved in site or autotransplanted. The use of carbon nanoparticles could help to reduce the incidence of permanent hypoparathyroidism and the parathyroid gland should be identified as much as possible to avoid being inadvertently resected.

\section{Abbreviations}

PG, parathyroid gland; PGA, parathyroid gland autotransplantation; APG, autotransplanted parathyroid gland; TT total thyroidectomy; BCND bilateral central lymph node dissection; PTH, parathyroid hormone; IEPG, inadvertently excisional parathyroid gland; ISPG, parathyroid gland in site; F, female; M male; BMI body mass index; ULND unilateral neck lymph node dissection (compartments II-V); BLND bilateral neck lymph node dissection (compartments II-V).

\section{Data Sharing Statement}

All data and code used and analyzed during the study are available from the corresponding author by reasonable request.

\section{Ethics Approval and Informed Consent}

The study was approved by the Medical Ethics Committee of Chengdu Third People's Hospital (No. 2019-S-90). This study was conducted in accordance with the Declaration of Helsinki.

\section{Consent for Publication}

All authors have reviewed the final version of the manuscript and are in agreement its' content and submission.

\section{Acknowledgments}

The authors thank the patients for their participation.

\section{Author Contributions}

All authors made a significant contribution to the work reported, whether that is in the conception, study design, execution, acquisition of data, analysis and interpretation, or in all these areas; took part in drafting, revising or critically reviewing the article; gave final approval of the version to be published; have agreed on the journal to which the article has been submitted; and agree to be accountable for all aspects of the work.

\section{Funding}

Bin Wang was supported by a nonprofit fund from CHINA HEALTH PROMOTION FOUNDATION. Jian Wu was supported by a grant from Scientific Research Fund of the Department of Science and Technology of Chengdu City (2015-HM01-00376-SF) and Science and Technology Program of Science \& Technology Department of Sichuan Province (2015JY0190). The funding bodies had no role in the conception of the study, in the collection, analysis, and interpretation of data, in writing the manuscript and in the approval of the publication.

\section{Disclosure}

Dr Bin Wang reports grants from CHINA HEALTH PROMOTION FOUNDATION, during the conduct of the study. Dr Jian Wu reports grants from Scientific Research Fund of the Department of Science and Technology of Chengdu City, grants from Science and Technology Program of Science \& Technology Department of Sichuan Province, during the conduct of the study. The authors report no conflicts of interest in this work.

\section{References}

1. Wells SA Jr., Asa SL, Dralle H, et al. Revised American thyroid association guidelines for the management of medullary thyroid carcinoma. Thyroid. 2015;25(6):567-610. doi:10.1089/thy.2014.0335

2. Haugen BR, Alexander EK, Bible KC, et al. 2015 American thyroid association management guidelines for adult patients with thyroid nodules and differentiated thyroid cancer: the American thyroid association guidelines task force on thyroid nodules and differentiated thyroid cancer. Thyroid. 2016;26(1):1-133.

3. Ponce de Leon-ballesteros G, Velazquez-Fernandez D, HernandezCalderon FJ, et al. Hypoparathyroidism after total thyroidectomy: importance of the intraoperative management of the parathyroid glands. World J Surg. 2019;43(7):1728-1735. doi:10.1007/s00268019-04987-z

4. Fama F, Cicciu M, Polito F, et al. Parathyroid autotransplantation during thyroid surgery: a novel technique using a cell culture nutrient solution. World $J$ Surg. 2017;41(2):457-463. doi:10.1007/s00268-016-3754-0

5. Giordano D, Valcavi R, Thompson GB, et al. Complications of central neck dissection in patients with papillary thyroid carcinoma: results of a study on 1087 patients and review of the literature. Thyroid. 2012;22 (9):911-917. doi:10.1089/thy.2012.0011

6. Lang BH, Ng SH, Lau LL, Cowling BJ, Wong KP, Wan KY. A systematic review and meta-analysis of prophylactic central neck dissection on short-term locoregional recurrence in papillary thyroid carcinoma after total thyroidectomy. Thyroid. 2013;23(9):1087-1098. doi: $10.1089 /$ thy.2012.0608

7. Viola D, Materazzi G, Valerio L, et al. Prophylactic central compartment lymph node dissection in papillary thyroid carcinoma: clinical implications derived from the first prospective randomized controlled single institution study. $J$ Clin Endocrinol Metab. 2015;100 (4):1316-1324. doi:10.1210/jc.2014-3825 
8. de Jong M, Nounou H, Rozalen Garcia V, et al. Children are at a high risk of hypocalcaemia and hypoparathyroidism after total thyroidectomy. J Pediatr Surg. 2020;55(7):1260-1264. doi:10.1016/ j.jpedsurg.2019.06.027

9. Paek SH, Lee YM, Min SY, Kim SW, Chung KW, Youn YK. Risk factors of hypoparathyroidism following total thyroidectomy for thyroid cancer. World J Surg. 2013;37(1):94-101. doi:10.1007/ s00268-012-1809-4

10. Su A, Gong Y, Wu W, Gong R, Li Z, Zhu J. Does the number of parathyroid glands autotransplanted affect the incidence of hypoparathyroidism and recovery of parathyroid function? Surgery. 2018;164 (1):124-129. doi:10.1016/j.surg.2017.12.025

11. Su A, Gong Y, Wu W, Gong R, Li Z, Zhu J. Effect of autotransplantation of a parathyroid gland on hypoparathyroidism after total thyroidectomy. Endocr Connect. 2018;7(2):286-294. doi:10.1530/ EC-17-0313

12. Bhattacharyya N, Fried MP. Assessment of the morbidity and complications of total thyroidectomy. Arch Otolaryngol Head Neck Surg. 2002;128(4):389-392. doi:10.1001/archotol.128.4.389

13. Palazzo FF, Sywak MS, Sidhu SB, Barraclough BH, Delbridge LW. Parathyroid autotransplantation during total thyroidectomy-does the number of glands transplanted affect outcome? World J Surg. 2005;29(5):629-631. doi:10.1007/s00268-005-7729-9

14. Zhu J, Tian W, Xu Z, et al. Expert consensus statement on parathyroid protection in thyroidectomy. Ann Transl Med. 2015;3(16):230.

15. Lorente-Poch L, Sancho J, Munoz JL, Gallego-Otaegui L, MartinezRuiz C, Sitges-Serra A. Failure of fragmented parathyroid gland autotransplantation to prevent permanent hypoparathyroidism after total thyroidectomy. Langenbecks Arch Surg. 2017;402(2):281-287. doi:10.1007/s00423-016-1548-3

16. Ahmed N, Aurangzeb M, Muslim M, Zarin M. Routine parathyroid autotransplantation during total thyroidectomy: a procedure with predictable outcome. J Pak Med Assoc. 2013;63(2):190-193.

17. Zedenius J, Wadstrom C, Delbridge L. Routine autotransplantation of at least one parathyroid gland during total thyroidectomy may reduce permanent hypoparathyroidism to zero. Aust $N$ Z J Surg. 1999;69 (11):794-797. doi:10.1046/j.1440-1622.1999.01697.x

18. Wang B, Zhu CR, Liu H, Wu J. The effectiveness of parathyroid gland autotransplantation in preserving parathyroid function during thyroid surgery for thyroid neoplasms: a meta-analysis. PLoS One. 2019;14(8):e0221173. doi:10.1371/journal.pone.0221173

19. Su A, Wang B, Gong Y, Gong R, Li Z, Zhu J. Risk factors of hypoparathyroidism following total thyroidectomy with central lymph node dissection. Medicine (Baltimore). 2017;96(39):e8162. doi:10.1097/MD.0000000000008162

20. Sitges-Serra A, Ruiz S, Girvent M, Manjon H, Duenas JP, Sancho JJ. Outcome of protracted hypoparathyroidism after total thyroidectomy. Br J Surg. 2010;97(11):1687-1695. doi:10.1002/bjs.7219

21. Su AP, Wei T, Liu F, Gong YP, Li ZH, Zhu JQ. Use of carbon nanoparticles to improve the dissection of lymph nodes and the identification of parathyroid glands during thyroidectomy for papillary thyroid cancer. Int J Clin Exp Med. 2016;9(10):19529-19536.

22. Senapati A, Young AE. Parathyroid autotransplantation. Br J Surg. 1990;77(10):1171-1174. doi:10.1002/bjs.1800771027

23. Kihara M, Yokomise H, Miyauchi A, Matsusaka K. Recovery of parathyroid function after total thyroidectomy. Surg Today. 2000;30 (4):333-338. doi:10.1007/s005950050596

24. Kihara M, Miyauchi A, Kontani K, Yamauchi A, Yokomise H. Recovery of parathyroid function after total thyroidectomy: long-term follow-up study. ANZ J Surg. 2005;75(7):532-536. doi:10.1111/j.1445-2197.2005.03435.x
25. White MG, James BC, Nocon C, et al. One-hour PTH after thyroidectomy predicts symptomatic hypocalcemia. J Surg Res. 2016;201 (2):473-479. doi:10.1016/j.jss.2015.11.028

26. Li Y, Jian WH, Guo ZM, Li QL, Lin SJ, Huang HY. A meta-analysis of carbon nanoparticles for identifying lymph nodes and protecting parathyroid glands during surgery. Otolaryngol Head Neck Surg. 2015;152(6):1007-1016. doi:10.1177/0194599815580765

27. Su AP, Wei T, Gong YP, Gong RX, Li ZH, Zhu JQ. Carbon nanoparticles improve lymph node dissection and parathyroid gland protection during thyroidectomy: a systematic review and meta-analysis. Int J Clin Exp Med. 2018;11(2):463-473.

28. Kikumori T, Imai T, Tanaka Y, Oiwa M, Mase T, Funahashi H. Parathyroid autotransplantation with total thyroidectomy for thyroid carcinoma: long-term follow-up of grafted parathyroid function. Surgery. 1999;125(5):504-508. doi:10.1016/S0039-6060(99)70201-1

29. El-Sharaky MI, Kahalil MR, Sharaky O, et al. Assessment of parathyroid autotransplantation for preservation of parathyroid function after total thyroidectomy. Head Neck. 2003;25(10):799-807. doi:10.1002/hed.10278

30. Lo CY, Tam SC. Parathyroid autotransplantation during thyroidectomy: documentation of graft function. Arch Surg. 2001;136 (12):1381-1385. doi:10.1001/archsurg.136.12.1381

31. Salander H, Tisell LE. Latent hypoparathyroidism in patients with autotransplanted parathyroid glands. Am J Surg. 1980;139 (3):385-388. doi:10.1016/0002-9610(80)90299-8

32. Song CM, Jung JH, Ji YB, Min HJ, Ahn YH, Tae K. Relationship between hypoparathyroidism and the number of parathyroid glands preserved during thyroidectomy. World J Surg Oncol. 2014;12 (1):200. doi:10.1186/1477-7819-12-200

33. Park I, Rhu J, Woo JW, Choi JH, Kim JS, Kim JH. Preserving parathyroid gland vasculature to reduce post-thyroidectomy hypocalcemia. World J Surg. 2016;40(6):1382-1389. doi:10.1007/ s00268-016-3423-3

34. Dekker A, Dunsford HA, Geyer SJ. The normal parathyroid gland at autopsy: the significance of stromal fat in adult patients. $J$ Pathol. 1979;128(3):127-132. doi:10.1002/path.1711280303

35. Dufour DR, Wilkerson SY. Factors related to parathyroid weight in normal persons. Arch Pathol Lab Med. 1983;107(4):167-172.

36. Pizzi H, Gladu J, Carpio L, Miao D, Goltzman D, Rabbani SA. Androgen regulation of parathyroid hormone-related peptide production in human prostate cancer cells. Endocrinology. 2003;144 (3):858-867. doi:10.1210/en.2002-220754

37. Sands NB, Payne RJ, Cote V, Hier MP, Black MJ, Tamilia M. Female gender as a risk factor for transient post-thyroidectomy hypocalcemia. Otolaryngol Head Neck Surg. 2011;145(4):561-564. doi:10.1177/0194599811414511

38. Karamanakos SN, Markou KB, Panagopoulos K, et al. Complications and risk factors related to the extent of surgery in thyroidectomy. Results from 2043 procedures. Hormones (Athens). 2010;9 (4):318-325. doi:10.14310/horm.2002.1283

39. Zhou HY, He JC, McHenry CR. Inadvertent parathyroidectomy: incidence, risk factors, and outcomes. J Surg Res. 2016;205 (1):70-75. doi:10.1016/j.jss.2016.06.019

40. Sitges-Serra A, Gallego-Otaegui L, Suarez S, Lorente-Poch L, Munne A, Sancho JJ. Inadvertent parathyroidectomy during total thyroidectomy and central neck dissection for papillary thyroid carcinoma. Surgery. 2017;161(3):712-719. doi:10.1016/j. surg.2016.08.021 


\section{Publish your work in this journal}

Cancer Management and Research is an international, peer-reviewed open access journal focusing on cancer research and the optimal use of preventative and integrated treatment interventions to achieve improved outcomes, enhanced survival and quality of life for the cancer patient.

The manuscript management system is completely online and includes a very quick and fair peer-review system, which is all easy to use. Visit http://www.dovepress.com/testimonials.php to read real quotes from published authors.

Submit your manuscript here: https://www.dovepress.com/cancer-management-and-research-journal 